\title{
Salmonella enterica subsp enterica
}

National Cancer Institute

\section{Source}

National Cancer Institute. Salmonella enterica subsp enterica. NCI Thesaurus. Code C86913.

A subspecies of aerobic, Gram negative, rod shaped bacteria assigned to the phylum Proteobacteria and the species Salmonella enterica. This subspecies does not produce acid from lactose, salicin, or galacturonate, will not hydrolyze gelatin, and exhibits no growth in the presence of KCN. S. enterica subsp. enterica is the causative agent of various types of salmonellosis. 